\title{
Hölder estimates of mild solutions for nonlocal SPDEs
}

\author{
Rongrong Tian' ${ }^{1}$ Liang Ding ${ }^{2}$, Jinlong $\mathrm{Wei}^{\mathrm{i}^{*}}$ (D) and Suyi Zheng ${ }^{3}$
}

"Correspondence:
weijinlong@zuel.edu.cn
${ }^{3}$ School of Statistics and
Mathematics, Zhongnan University
of Economics and Law, Wuhan,
China
Full list of author information is
available at the end of the article

available at the end of the article

\begin{abstract}
We consider nonlocal PDEs driven by additive white noises on $\mathbb{R}^{d}$. For $L^{q}$ integrable coefficients, we derive the existence and uniqueness, as well as Hölder continuity, of mild solutions. Precisely speaking, the unique mild solution is almost surely Hölder continuous with Hölder index $0<\theta<(1 / 2-d /(q \alpha))(1 \wedge \alpha)$. Moreover, we show that any order $\gamma(<q)$ moment of Hölder normal for $u$ on every bounded domain of $\mathbb{R}_{+} \times \mathbb{R}^{d}$ is finite.
\end{abstract}

MSC: $60 \mathrm{H} 15 ; 35 \mathrm{R} 60$

Keywords: Stochastic partial differential equation; Hölder-estimates

\section{Introduction}

Let $\left(\Omega, \mathcal{F},\left\{\mathcal{F}_{t}\right\}_{t \geq 0}, \mathbb{P}\right)$ be a filtered probability space that satisfies the usual hypotheses of completeness and right continuity. $\left\{W_{t}\right\}_{t \geq 0}$ is a one-dimensional standard Wiener process on $\left(\Omega, \mathcal{F},\left\{\mathcal{F}_{t}\right\}_{t \geq 0}, \mathbb{P}\right)$. In this paper, we are concerned with the Hölder-estimates of mild solutions for the following nonlocal stochastic partial differential equations (SPDEs for short):

$$
\left\{\begin{array}{l}
d u(t, x)+(-\Delta)^{\frac{\alpha}{2}} u(t, x) d t=h(t, x) d t+f(t, x) d W_{t}, \quad t>0, x \in \mathbb{R}^{d} \\
\left.u(t, x)\right|_{t=0}=0, \quad x \in \mathbb{R}^{d}
\end{array}\right.
$$

where $\alpha \in(0,2],(-\Delta)^{\frac{\alpha}{2}}$ is the fractional Laplacian on $\mathbb{R}^{d}$.

When $\alpha=2$, these SPDEs have been studied widely. $W^{k, 2}$-theory was well established by Pardoux [15] and Rozovskii [16]. A more general $W^{2, q}$-theory was founded by Krylov [9-11] for $2 \leq q<\infty$. Krylov's result was then generalized by Denis, Matoussi, and Stoica [5] for $q=\infty$ to nonlinear SPDEs.

There is also some Hölder estimates for solutions of (1.1) when $\alpha=2$. As $f(t, \cdot)$ belongs to $L^{q}$ with large enough $q$ (or $q=\infty$ ), $h$ vanishes and $\mathbb{R}^{d}$ is replaced by a bounded domain (with smooth boundary), the space and time Hölder estimates have been discussed by Kuksin, Nadirashvili, and Piatnitski $[12,13]$. This result was further developed by Kim [8] for general Hölder estimates for generalized solutions with $L_{p}\left(L_{q}\right)$ coefficients. Using a different philosophy, Hsu, Wang, and Wang [6] discussed (1.1) with $\alpha=2$ for general $f$ ( $u$ dependent). By applying a stochastic De Giorgi iteration technique, they built the Hölder estimates for weak solutions on $[T, 2 T] \times \mathbb{R}^{d}(T>0)$. Recently, by using the heat

(c) The Author(s) 2019. This article is distributed under the terms of the Creative Commons Attribution 4.0 International License (http://creativecommons.org/licenses/by/4.0/), which permits unrestricted use, distribution, and reproduction in any medium, provided you give appropriate credit to the original author(s) and the source, provide a link to the Creative Commons license, and indicate if changes were made. 
kernel estimate technique, Wei, Duan, and Lv [18] also derived the Hölder estimates for stochastic transport-diffusion equations driven by Lévy noises.

When $\alpha \in(0,2)$, Chang and Lee [4], Kim and Kim [7] studied the $L^{q}(2 \leq q<\infty)$ theory for SPDE (1.1). When $f$ is bounded measurable, by constructing stochastic BMO and Morrey-Campanato spaces, Lv et al. [14] established the BMO and Hölder estimates for solutions.

However, as far as we know, there have been very few papers dealing with the Hölder estimates for (1.1) with $L^{q}$ coefficients. In this paper, we will fill this gap and derive the Hölder estimates for mild solutions. Here the mild solution of (1.1) is defined as follows.

Definition 1.1 Let $\alpha \in(0,2)$ and $P_{t}$ denote the forward heat semigroup generated by negative fractional Laplacian $-(-\Delta)^{\alpha / 2}$. Suppose that $u$ is given by

$$
u(t, x)=\int_{0}^{t} P_{t-r} h(r, \cdot)(x) d r+\int_{0}^{t} P_{t-r} f(r, \cdot)(x) d W_{r} .
$$

We call $u$ a mild solution of $(1.1)$ if $u \in L_{\text {loc }}^{\infty}\left([0, \infty) ; L^{\infty}\left(\mathbb{R}^{d} ; L^{2}(\Omega)\right)\right)$ which is $\mathcal{F}_{t}$-adapted and as a family of $L^{2}(\Omega, \mathcal{F}, \mathbb{P})$-valued random variables is continuous.

Remark 1.1 Let $p(t, x, y)$ be the transition density of symmetric $\alpha$-stable process, then $p(t, x, y)=p(t, x-y)$ and

$$
P_{t} \varphi(x)=\int_{\mathbb{R}^{d}} p(t, x-y) \varphi(y) d y, \quad \varphi \in L^{q}\left(\mathbb{R}^{d}\right), q>1
$$

Moreover, $p(t, \cdot)$ is smooth for $t>0$ and from [4, Lemma 2.2] (also see [3, 19, 20]), we have the following estimates:

$$
\begin{aligned}
& p(t, x-y) \approx \frac{t}{|y-x|^{d+\alpha}} \wedge t^{-\frac{d}{\alpha}}, \\
& \left|\nabla_{x} p(t, x-y)\right| \approx|y-x|\left(\frac{t}{|y-x|^{d+2+\alpha}} \wedge t^{-\frac{d+2}{\alpha}}\right) .
\end{aligned}
$$

For every $t>0$, by the scaling property, then $p(t, x-y)=t^{-\frac{d}{\alpha}} p\left(1,(x-y) t^{-1 / \alpha}\right)$, which implies

$$
\left|\partial_{t} p(t, x-y)\right| \leq C\left(\frac{1}{|y-x|^{d+\alpha}} \wedge t^{-\frac{d+\alpha}{\alpha}}\right)
$$

Our main result is the following.

Theorem 1.1 Let us consider the nonlocal SPDE (1.1) associated with $\alpha \in(0,2)$. We suppose that $q>2 d / \alpha \vee 2, f, h \in L_{\text {loc }}^{\infty}\left(\mathbb{R}_{+} ; L^{q}\left(\mathbb{R}^{d} \times \Omega\right)\right)$ which are $\mathcal{F}_{t}$-adapted. Let us set $\vartheta=(1 / 2-d /(q \alpha))(1 \wedge \alpha)$. Then there is a mild solution $u$ of $(1.1)$ and $u \in L_{\mathrm{loc}}^{\infty}\left(\mathbb{R}_{+} ; L^{\infty}\left(\mathbb{R}^{d} ;\right.\right.$ $\left.\left.L^{q}(\Omega)\right)\right)$.

In addition, if $q>4(d+1) /(1 \wedge \alpha), u \in \mathcal{C}^{\vartheta-}\left([0, t] \times \mathbb{R}^{d} ; L^{q}(\Omega)\right) \cap L^{q-}\left(\Omega ; \mathcal{C}_{\text {loc }}^{\vartheta-}\left(\mathbb{R}_{+} \times \mathbb{R}^{d}\right)\right)$ for every $0<t<\infty$. Moreover, for every $t>0$, every $0<\theta<\vartheta$, every bounded domain $Q \subset$ $\mathbb{R}^{d}$, every $0<\gamma<q$, there exist two positive constants $C(q, \alpha, d, \theta, t)$ and $C(q, \alpha, d, \gamma, \theta, t, Q)$ 
(independent of $h$ and $f$ ) such that

$$
\begin{aligned}
\|u\|_{\mathcal{C}^{\theta}\left([0, t] \times \mathbb{R}^{d} ; L^{q}(\Omega)\right)} \leq & C(q, \alpha, d, \theta, t)\left[\|h\|_{L^{\infty}\left([0, t] ; L^{q}\left(\mathbb{R}^{d} \times \Omega\right)\right)}\right. \\
& \left.+\|f\|_{L^{\infty}\left([0, t] ; L^{q}\left(\mathbb{R}^{d} \times \Omega\right)\right)}\right]
\end{aligned}
$$

and

$$
\begin{aligned}
\mathbb{E}\|u\|_{\mathcal{C}^{\theta}([0, t] \times \bar{Q})}^{\gamma} \leq & C(q, \alpha, d, \gamma, \theta, t, Q)\left[\|h\|_{L^{\infty}\left([0, t] ; L^{q}\left(\mathbb{R}^{d} \times \Omega\right)\right)}\right. \\
& \left.+\|f\|_{L^{\infty}\left([0, t] ; L^{q}\left(\mathbb{R}^{d} \times \Omega\right)\right)}\right]^{\gamma},
\end{aligned}
$$

where

$$
\mathcal{C}^{\vartheta-}\left([0, t] \times \mathbb{R}^{d} ; L^{q}(\Omega)\right):=\bigcap_{0<\theta<\vartheta} \mathcal{C}^{\theta}\left([0, t] \times \mathbb{R}^{d} ; L^{q}(\Omega)\right)
$$

and

$$
L^{q-}\left(\Omega ; \mathcal{C}_{\mathrm{loc}}^{\vartheta-}\left(\mathbb{R}_{+} \times \mathbb{R}^{d}\right)\right):=\bigcap_{1 \leq p<q} L^{p}\left(\Omega ; \bigcap_{0<\theta<\vartheta} \mathcal{C}_{\mathrm{loc}}^{\theta}\left(\mathbb{R}_{+} \times \mathbb{R}^{d}\right)\right) .
$$

Remark 1.2 (i) In [4] Chang and Lee discussed (1.1); under the assumptions that $h \in$ $H_{q}^{k}\left(T, \mathbb{R}^{d}\right), f \in H_{q}^{k+\frac{\alpha}{2}+\delta}\left(T, \mathbb{R}^{d}\right)(T>0$ is a given real number, $0<\delta<\alpha / 2)$, they founded the $H_{q}^{k+\alpha}$ theory of solutions on $\mathbb{R}^{d}$. As a direct consequence, if $k=0$ and $q \alpha>d$, the Hölder estimate for solutions in space variable satisfies

$$
\mathbb{E} \int_{0}^{t}\|u(s)\|_{\mathcal{C}^{\theta}\left(\mathbb{R}^{d}\right)}^{\gamma} d s<\infty, \quad t>0
$$

where $\theta$ is given by the Sobolev imbedding theorem. Different from [4], $L^{\infty}\left(L^{q}\right)$ integrability in space and time variables is enough to ensure the Hölder continuity of solutions in space and time variables.

(ii) Our main idea comes from [13]. In [13], Kuksin, Nadirashvili, and Piatnitski argued (1.1) with $\alpha=2$ on a bounded domain. By estimating the tail probability, they gained the space and time Hölder estimates. Here, we study (1.1) on $\mathbb{R}^{d}$ with $\alpha \in(0,2)$. By using the techniques developed in [13], we gain the space and time Hölder estimates on every bounded domain.

This paper is organized as follows. In Sect. 2, we present some useful lemmas, and Sect. 3 is devoted to giving the proof details.

Notations $a \wedge b=\min \{a, b\}, a \vee b=\max \{a, b\} . \mathbb{R}_{+}=\{r \in \mathbb{R}, r \geq 0\}$. The letter $C$ will mean a positive constant whose values may change in different places. For a parameter or a function $\varrho, C(\varrho)$ means the constant is only dependent on $\varrho . \mathbb{N}$ is the set of natural numbers, and $\mathbb{Z}$ denotes the set of integral numbers. Let $Q \subset \mathbb{R}^{k}(k \in \mathbb{N})$ be a bounded domain. For $0<\theta<1$, we define $\mathcal{C}^{\theta}(\bar{Q})$ to be the set of all continuous functions $u$ on $Q$ such that

$$
\|u\|_{\mathcal{C}^{\theta}(\bar{Q})}:=\sup _{x \in \bar{Q}}|u(x)|+\sup _{x, y \in \bar{Q}, x \neq y} \frac{|u(x)-u(y)|}{|x-y|^{\theta}}<\infty .
$$




\section{Useful lemmas}

Lemma 2.1 Let $\rho_{0} \in L^{q}\left(\mathbb{R}^{d} \times \Omega\right)$. Consider the Cauchy problem

$$
\partial_{t} \rho(t, x)+(-\Delta)^{\frac{\alpha}{2}} \rho(t, x)=0, \quad t>0, x \in \mathbb{R}^{d},\left.\rho(t, x)\right|_{t=0}=\rho_{0}(x) .
$$

Then, for any $0<\beta<1$, the unique mild solution (given by (1.3) if one replaces $\varphi$ by $\rho_{0}$ ) of (2.1) meets the following estimates:

$$
\|\rho(t)\|_{\mathcal{C}^{\beta}\left(\mathbb{R}^{d}\right)} \leq C t^{-\frac{\beta}{\alpha}-\frac{d}{q \alpha}}\left\|\rho_{0}\right\|_{L^{q}\left(\mathbb{R}^{d}\right)}, \quad \mathbb{P} \text {-a.s. } \omega \in \Omega
$$

and

$$
|\rho(t+\delta, x)-\rho(t, x)| \leq C t^{-\beta-\frac{d}{q \alpha}} \delta^{\beta}\left\|\rho_{0}\right\|_{L^{q\left(\mathbb{R}^{d}\right)}}, \quad \mathbb{P} \text {-a.s. } \omega \in \Omega
$$

Proof Obviously, the unique mild solution $\rho$ of (2.1) can be represented by (1.3) if one replaces $\varphi$ by $\rho_{0}$. Hence, for any $t>0$,

$$
\begin{aligned}
|\rho(t, x)| & \leq \int_{\mathbb{R}^{d}}\left|p(t, x-y) \rho_{0}(y)\right| d y \\
& \leq\left[\int_{\mathbb{R}^{d}}|p(t, y)|^{\frac{q}{q-1}} d y\right]^{\frac{q-1}{q}}\left\|\rho_{0}\right\|_{L^{q\left(\mathbb{R}^{d}\right)}}, \quad \mathbb{P} \text {-a.s. } \omega \in \Omega .
\end{aligned}
$$

According to (1.4), then

$$
\begin{aligned}
& \int_{\mathbb{R}^{d}}|p(t, y)|^{\frac{q}{q-1}} d y \leq C\left[\int_{|y| \geq t \frac{1}{\alpha}}\left|\frac{t}{|y|^{d+\alpha}} \wedge t^{-\frac{d}{\alpha}}\right|^{\frac{q}{q-1}} d y+\int_{|y|<t \frac{1}{\alpha}}\left|\frac{t}{|y|^{d+\alpha}} \wedge t^{-\frac{d}{\alpha}}\right|^{\frac{q}{q-1}} d y\right] \\
& \leq C\left[\int_{|y| \geq t \frac{1}{\alpha}}\left|\frac{t}{|y|^{d+\alpha}}\right|^{\frac{q}{q-1}} d y+\int_{|y|<t^{\frac{1}{\alpha}}}\left(t^{-\frac{d}{\alpha}}\right)^{\frac{q}{q-1}} d y\right] \\
& =C\left[t^{\frac{q}{q-1}} \int_{|y| \geq t^{\frac{1}{\alpha}}}|y|^{-\frac{q(d+\alpha)}{q-1}} d y+t^{-\frac{d q}{(q-1) \alpha}} \int_{|y|<t^{\frac{1}{\alpha}}} d y\right] \\
& \leq C\left[\left.t^{\frac{q}{q-1}}|y|^{-\frac{q(d+\alpha)}{q-1}+d}\right|_{y=t^{\frac{1}{\alpha}}}+\left.t^{-\frac{d q}{(q-1) \alpha}}|y|^{d}\right|_{y=t^{\frac{1}{\alpha}}}\right] \\
& =C t^{-\frac{d}{(q-1) \alpha}} \text {. }
\end{aligned}
$$

Combining (2.4) and (2.5), then

$$
|\rho(t, x)| \leq \int_{\mathbb{R}^{d}}\left|p(t, x-y) \rho_{0}(y)\right| d y \leq C t^{-\frac{d}{q \alpha}}\left\|\rho_{0}\right\|_{L^{q}\left(\mathbb{R}^{d}\right)}, \quad \mathbb{P} \text {-a.s. } \omega \in \Omega .
$$

Let us calculate $\nabla \rho$ and $\partial_{t} \rho$. For $1 \leq i \leq d$, we manipulate that

$$
\partial_{x_{i}} \rho(t, x)=\int_{\mathbb{R}^{d}} \partial_{x_{i}} p(t, x-y) \rho_{0}(y) d y
$$

which suggests that

$$
|\nabla \rho(t, x)| \leq\left[\int_{\mathbb{R}^{d}}|\nabla p(t, y)|^{\frac{q}{q-1}} d y\right]^{\frac{q-1}{q}}\left\|\rho_{0}\right\|_{L^{q}\left(\mathbb{R}^{d}\right)}, \quad \mathbb{P} \text {-a.s. } \omega \in \Omega .
$$


By virtue of (1.4) and analogue calculations for (2.5) imply that

$$
\begin{aligned}
& \int_{\mathbb{R}^{d}}|\nabla p(t, y)|^{\frac{q}{q-1}} d y \\
& \leq C\left[\left.\int_{|y| \geq t^{\frac{1}{\alpha}}}|y|\left(\frac{t}{|y|^{d+2+\alpha}} \wedge t^{-\frac{d+2}{\alpha}}\right)\right|^{\frac{q}{q-1}} d y+\int_{|y|<t \frac{1}{\alpha}}|| y\left|\left(\frac{t}{|y|^{d+2+\alpha}} \wedge t^{-\frac{d+2}{\alpha}}\right)\right|^{\frac{q}{q-1}} d y\right] \\
& \leq C\left[\int_{|y| \geq t^{\frac{1}{\alpha}}} t^{\frac{q}{q-1}}|y|^{-\frac{q(d+1+\alpha)}{q-1}} d y+\int_{|y|<t^{\frac{1}{\alpha}}}|y|^{\frac{q}{q-1}} t^{-\frac{q(d+2)}{(q-1) \alpha}} d y\right] \\
& \leq C\left[\left.t^{\frac{q}{q-1}}|y|^{-\frac{q(d+1+\alpha)}{q-1}+d}\right|_{y=t^{\frac{1}{\alpha}}}+\left.t^{-\frac{(d+2) q}{(q-1) \alpha}}|y|^{d+\frac{q}{q-1}}\right|_{y=t \frac{1}{\alpha}}\right] \\
& =C t^{-\frac{d+q}{(q-1) \alpha}} \text {. }
\end{aligned}
$$

Therefore, one arrives at

$$
|\nabla \rho(t, x)| \leq C t^{-\frac{d}{q \alpha}-\frac{1}{\alpha}}\left\|\rho_{0}\right\|_{L^{q}\left(\mathbb{R}^{d}\right)}, \quad \mathbb{P} \text {-a.s. } \omega \in \Omega .
$$

Applying the interpolation inequality

$$
\|\rho(t)\|_{\mathcal{C}^{\beta}\left(\mathbb{R}^{d}\right)} \leq C\|\rho(t)\|_{L^{\infty}\left(\mathbb{R}^{d}\right)}^{1-\beta}\|\rho(t)\|_{\mathcal{C}^{1}\left(\mathbb{R}^{d}\right)}^{\beta}
$$

to (2.6) and (2.7), (2.2) holds true.

Repeating the above calculations, and by virtue of (1.5), one derives that

$$
\left|\partial_{t} \rho(t, x)\right| \leq C t^{-\frac{d}{q \alpha}-1}\left\|\rho_{0}\right\|_{L q\left(\mathbb{R}^{d}\right)}, \quad \mathbb{P} \text {-a.s. } \omega \in \Omega .
$$

The interpolation inequality (2.8) uses (2.6) and (2.9), for every $t_{2}>t_{1}>0$, we get

$$
\|\rho(\cdot, x)\|_{\mathcal{C}^{\beta}\left(\left[t_{1}, t_{2}\right]\right)} \leq C t_{1}^{-\beta-\frac{d}{q \alpha}}\left\|\rho_{0}\right\|_{L^{q\left(\mathbb{R}^{d}\right)}}, \quad \mathbb{P} \text {-a.s. } \omega \in \Omega .
$$

From (2.10), inequality (2.3) is legitimate, and we finish the proof.

Lemma 2.2 (Minkowski inequality [17]) Assume that $\left(S_{1}, \mathcal{F}_{1}, \mu_{1}\right)$ and $\left(S_{2}, \mathcal{F}_{2}, \mu_{2}\right)$ are two measure spaces and that $G: S_{1} \times S_{2} \rightarrow \mathbb{R}$ is measurable. For given real numbers $1 \leq p_{1} \leq$ $p_{2}$, we also assume that $G \in L^{p_{1}}\left(S_{1} ; L^{p_{2}}\left(S_{2}\right)\right)$. Then $G \in L^{p_{2}}\left(S_{2} ; L^{p_{1}}\left(S_{1}\right)\right)$ and

$$
\begin{aligned}
& {\left[\int_{S_{2}}\left(\int_{S_{1}}|G(x, y)|^{p_{1}} \mu_{1}(d x)\right)^{\frac{p_{2}}{p_{1}}} \mu_{2}(d y)\right]^{\frac{1}{p_{2}}}} \\
& \quad \leq\left[\int_{S_{1}}\left(\int_{S_{2}}|G(x, y)|^{p_{2}} \mu_{2}(d y)\right)^{\frac{p_{1}}{p_{2}}} \mu_{1}(d x)\right]^{\frac{1}{p_{1}}} .
\end{aligned}
$$

The next lemmas will play an important role in estimating stochastic integrals.

Lemma 2.3 (Interpolation inequality) Suppose that $1 \leq p_{1}<p_{2} \leq \infty$. Let $E$ be a Banach space and $F$ be a linear operator from $L^{p_{1}}(\Omega ; E)+L^{p_{2}}(\Omega ; E)$ into the space $L^{p_{1}}(\Omega)+L^{p_{2}}(\Omega)$. If $F$ is bounded from $L^{p_{1}}(\Omega ; E)$ into $L^{p_{1}}(\Omega)$ and also bounded from $L^{p_{2}}(\Omega ; E)$ into $L^{p_{2}}(\Omega)$, then $F$ is bounded from $L^{p_{3}}(\Omega ; E)$ into $L^{p_{3}}(\Omega)$ for every $p_{1} \leq p_{3} \leq p_{2}$. 
Proof When $E=\mathbb{R}$, this result can be recovered from the Marcinkiewicz interpolation theorem [1, Theorem 2.58]. For a general Banach space $E$, what we should do is to replace $\delta_{u}(\tau)\left[1\right.$, pp. 56-57] by $\delta_{\|u\|_{E}}(\tau)$, and then the lemma is proved.

Lemma 2.4 Let $\mathcal{F}$ be given in the introduction, that $g$ be an $\mathcal{F} \times \mathcal{B}\left(\mathbb{R}_{+}\right) \times \mathcal{B}\left(\mathbb{R}_{+}\right) \times \mathcal{B}\left(\mathbb{R}^{d}\right)$ measurable function. Suppose that $\left\{M_{t}(x)\right\}_{t \geq 0}$ is a Brownian type integral of the form

$$
M_{t}(x)=\int_{0}^{t} g(t, r, x) d W_{r}, g(\cdot, r, \cdot) \quad \text { is } \mathcal{F}_{r} \text {-measurable. }
$$

Suppose that $q \geq 2$ and

$$
\mathbb{E}\left[\int_{0}^{t}|g(t, r, x)|^{2} d r\right]^{\frac{q}{2}}<\infty \quad \text { for almost everywhere } x \in \mathbb{R}^{d} .
$$

There exists a positive constant $C(q)>0$, which is independent of $x$, such that for each $t \geq 0$,

$$
\mathbb{E}\left[\left|M_{t}(x)\right|^{q}\right] \leq C(q) \mathbb{E}\left[\int_{0}^{t}|g(t, r, x)|^{2} d r\right]^{\frac{q}{2}} .
$$

Proof First, we assume that $F$ has the following form:

$$
g(t, r, x)=\sum_{j=1}^{k} g_{j}(t, x) 1_{\left(t_{j-1}, t_{j}\right]}(r)
$$

where $k \in \mathbb{N}, g_{j}$ are $\left(\Omega \times \mathbb{R}_{+} \times \mathbb{R}^{d} ; \mathcal{F}_{t_{j-1}} \times \mathcal{B}\left(\mathbb{R}_{+}\right) \times \mathcal{B}\left(\mathbb{R}^{d}\right)\right)$-measurable, and $0=t_{0}<t_{1}<$ $t_{2}<\cdots<t_{k}=t$.

For $q=2$, by using the Itô isometry, we obtain

$$
\mathbb{E}\left|M_{t}(x)\right|^{2}=\mathbb{E}\left|\sum_{j=1}^{k}\left(W_{t_{j}}-W_{t_{j-1}}\right) g_{j}(t, x)\right|^{2}=\mathbb{E} \int_{0}^{t}|g(t, r, x)|^{2} d r .
$$

For $q=4$, according to Burkholder's inequality [2, Theorem 4.4.21], we also have

$$
\mathbb{E}\left|M_{t}(x)\right|^{4}=\mathbb{E}\left|\sum_{j=1}^{k}\left(W_{t_{j}}-W_{t_{j-1}}\right) g_{j}(t, x)\right|^{4} \leq C \mathbb{E}\left[\int_{0}^{t}|g(t, r, x)|^{2} d r\right]^{2} .
$$

From (2.15) and (2.16), for every $t>0$, the linear operator

$$
F: g \rightarrow \int_{0}^{t} g(t, r, x) d W_{r}
$$

is bounded from $L^{2}\left(\Omega ; L^{2}(0, t)\right)$ into $L^{2}(\Omega)$ and also bounded from $L^{4}\left(\Omega ; L^{2}(0, t)\right)$ into $L^{4}(\Omega)$. According to Lemma 2.3, $F$ is bounded from $L^{q}\left(\Omega ; L^{2}(0, t)\right)$ into $L^{q}(\Omega)$ for every $2 \leq q \leq 4$, i.e., (2.13) holds true if $g$ has the form (2.14). Observing that the functions which meet condition (2.13) can be approximated by the step functions, we thus complete the proof for $q \in[2,4]$. 
Analogously, for every even number and every step function of the form (2.14), one can prove that (2.16) holds. In view of Lemma 2.3, one derives an inequality of (2.13) for every $q>4$. Then, by an approximating argument, we accomplish the proof.

Remark 2.1 When $g(t, r, x)$ is a deterministic function, the Marcinkiewicz interpolation inequality is not needed. Indeed, an $L^{q}$ type interpolation inequality is enough.

Lemma 2.5 ([13, Lemma 4]) Let a function $g$ satisfy the estimate

$$
\underset{J}{\operatorname{osc}} g=\sup _{x, y \in J}|g(x)-g(y)| \leq \kappa_{n}
$$

in any small cube $J$ which is a mesh of the grid $2^{-n} \mathbb{Z}^{d+1}$, i.e., in any $J=2^{-n} j+\left[0,2^{-n}\right]^{d+1}$, where $j \in \mathbb{Z}^{d+1}$. Then, for any $\Delta \in \mathbb{R}^{d+1}$, one has

$$
|g(y+\Delta)-g(y)| \leq 2 \kappa_{\left[\log _{2}(1 / \Delta)\right]}
$$

where [.] stands for the integer part.

\section{Proof of Theorem 1.1}

Proof The existence result follows by using the explicit formula

$$
u(t, x)=\int_{0}^{t} \int_{\mathbb{R}^{d}} p(t-r, x-y) h(r, y) d y d r+\int_{0}^{t} \int_{\mathbb{R}^{d}} p(t-r, x-y) f(r, y) d y d W_{r}
$$

where $p(t, x-y)$ fulfills (1.4) and (1.5). By this obvious representation, to prove $u$ is a mild solution, we need to show $u \in L_{\text {loc }}^{\infty}\left(\mathbb{R}_{+} ; L^{\infty}\left(\mathbb{R}^{d} ; L^{2}(\Omega)\right)\right)$. Now let us verify that $u \in L_{\mathrm{loc}}^{\infty}\left(\mathbb{R}_{+} ; L^{\infty}\left(\mathbb{R}^{d} ; L^{q}(\Omega)\right)\right)$.

If one uses Lemma 2.4 for given $q \geq 2$, then

$$
\begin{aligned}
\mathbb{E}|u(t, x)|^{q} \leq & C(q) \mathbb{E}\left|\int_{0}^{t} \int_{\mathbb{R}^{d}} p(t-r, x-z) h(r, z) d z d r\right|^{q} \\
& +C(q) \mathbb{E}\left[\int_{0}^{t}\left|\int_{\mathbb{R}^{d}} p(t-r, x-z) f(r, z) d z\right|^{2} d r\right]^{\frac{q}{2}} .
\end{aligned}
$$

With the aid of Lemma 2.2 and the Hölder inequality, we arrive at

$$
\begin{aligned}
\mathbb{E}|u(t, x)|^{q} \leq & C(q)\left|\int_{0}^{t} \int_{\mathbb{R}^{d}} p(t-r, x-z)\left[\mathbb{E}|h(r, z)|^{q}\right]^{\frac{1}{q}} d z d r\right|^{q} \\
& +C(q) \mathbb{E}\left[\int_{0}^{t}\left|\int_{\mathbb{R}^{d}} p(t-r, x-z)\left[\mathbb{E}|f(r, z)|^{q}\right]^{\frac{1}{q}} d z\right|^{2} d r\right]^{\frac{q}{2}} \\
\leq & C(q)\left|\int_{0}^{t}\left[\int_{\mathbb{R}^{d}}|p(r, y)|^{\frac{q}{q-1}} d y\right]^{\frac{q-1}{q}} d r\right|^{q} \sup _{0 \leq r \leq t} \mathbb{E} \int_{\mathbb{R}^{d}}|h(r, z)|^{q} d z \\
& +C(q)\left|\int_{0}^{t}\left[\int_{\mathbb{R}^{d}}|p(r, y)|^{\frac{q}{q-1}} d y\right]^{\frac{2(q-1)}{q}} d r\right|_{0 \leq r \leq t}^{\frac{q}{2}} \sup _{\mathbb{R}^{d}}|f(r, z)|^{q} d z .
\end{aligned}
$$


By using inequality (2.5), one derives

$$
\begin{aligned}
\mathbb{E}|u(t, x)|^{q} \leq & C(q)\left|\int_{0}^{t} r^{-\frac{d}{q \alpha}} d r\right|^{q}\|h\|_{L^{\infty}\left([0, t] ; L^{q}\left(\mathbb{R}^{d} \times \Omega\right)\right)}^{q} \\
& +C(q)\left|\int_{0}^{t} r^{-\frac{2 d}{q \alpha}} d r\right|^{\frac{q}{2}}\|f\|_{L^{\infty}\left([0, t] ; L^{q}\left(\mathbb{R}^{d} \times \Omega\right)\right)}^{q} .
\end{aligned}
$$

Observing that $q \alpha>2 d$, therefore

$$
\mathbb{E}|u(t, x)|^{q} \leq C(q)\left(1+t^{q-\frac{d}{\alpha}}\right)\left[\|h\|_{L^{\infty}\left([0, t] ; L^{q}\left(\mathbb{R}^{d} \times \Omega\right)\right)}^{q}+\|f\|_{L^{\infty}\left([0, t] ; L^{q}\left(\mathbb{R}^{d} \times \Omega\right)\right)}^{q}\right] .
$$

Now let us consider point-wise estimates for mild solutions of (1.1). By the scaling transformations on space and time variables, to prove (1.6) and (1.7) are true for $u$, it is sufficient to show that $u$ meets $(1.6)$ and $(1.7)$ on $[0,1] \times \mathbb{R}^{d}$ and $[0,1]^{d+1}$, respectively. Initially, let us check (1.6).

For every $x_{1}, x_{2} \in \mathbb{R}^{d}, t>0$,

$$
\begin{aligned}
u\left(t, x_{1}\right)-u\left(t, x_{2}\right)= & \int_{0}^{t}\left[P_{t-r} h\left(r, x_{1}\right)-P_{t-r} h\left(r, x_{2}\right)\right] d r \\
& +\int_{0}^{t}\left[P_{t-r} f\left(r, x_{1}\right)-P_{t-r} f\left(r, x_{2}\right)\right] d W_{r} .
\end{aligned}
$$

According to (2.13), one derives that

$$
\begin{aligned}
\mathbb{E}\left|u\left(t, x_{1}\right)-u\left(t, x_{2}\right)\right|^{q} \leq & C(q)\left\{\mathbb{E}\left|\int_{0}^{t}\left[P_{t-r} h\left(r, x_{1}\right)-P_{t-r} h\left(r, x_{2}\right)\right] d r\right|^{q}\right. \\
& \left.+\mathbb{E}\left|\int_{0}^{t}\left[P_{t-r} f\left(r, x_{1}\right)-P_{t-r} f\left(r, x_{2}\right)\right]^{2} d r\right|^{\frac{q}{2}}\right\} .
\end{aligned}
$$

Let $0<\beta<(1 / 2-d /(q \alpha))(1 \wedge \alpha)$ be a real number. In view of Lemma 2.1 (2.2) and Lemma 2.2 (2.11), from (3.2), one concludes that

$$
\begin{aligned}
\mathbb{E}\left|u\left(t, x_{1}\right)-u\left(t, x_{2}\right)\right|^{q} & \\
\leq & C(q)\left|x_{1}-x_{2}\right|^{q \beta}\|h\|_{L^{\infty}\left([0, t] ; L^{q}\left(\mathbb{R}^{d} \times \Omega\right)\right)}^{q}\left|\int_{0}^{t} r^{-\frac{\beta}{\alpha}-\frac{d}{q \alpha}} d r\right|^{q} \\
& +C(q)\left|x_{1}-x_{2}\right|^{q \beta}\|f\|_{L^{\infty}\left([0, t] ; L^{q}\left(\mathbb{R}^{d} \times \Omega\right)\right)}^{q}\left|\int_{0}^{t} r^{-\frac{2 \beta}{\alpha}-\frac{2 d}{q \alpha}} d r\right|^{\frac{q}{2}} \\
\leq & C(q, \alpha, d, \beta, t)\left|x_{1}-x_{2}\right|^{q \beta}\left[\|h\|_{L^{\infty}\left([0, t] ; L^{q}\left(\mathbb{R}^{d} \times \Omega\right)\right)}^{q}+\|f\|_{L^{\infty}\left([0, t] ; L^{q}\left(\mathbb{R}^{d} \times \Omega\right)\right)}^{q}\right] .
\end{aligned}
$$

Similarly, for every $t>0, \delta>0$, one can define

$$
\begin{aligned}
& u(t+\delta, x)-u(t, x) \\
& =\int_{0}^{t+\delta} P_{t+\delta-r} h(r, x) d r-\int_{0}^{t} P_{t-r} h(r, x) d r \\
& \quad+\int_{0}^{t+\delta} P_{t+\delta-r} f(r, x) d W_{r}-\int_{0}^{t} P_{t-r} f(r, x) d W_{r}
\end{aligned}
$$




$$
\begin{aligned}
= & \int_{t}^{t+\delta} P_{t+\delta-r} h(r, x) d r+\int_{0}^{t}\left[P_{t+\delta-r} h(r, x)-P_{t-r} h(r, x)\right] d r \\
& +\int_{t}^{t+\delta} P_{t+\delta-r} f(r, x) d W_{r}+\int_{0}^{t}\left[P_{t+\delta-r} f(r, x)-P_{t-r} f(r, x)\right] d W_{r} \\
= & : J_{1}+J_{2}+J_{3}+J_{4} .
\end{aligned}
$$

Let us estimate $J_{1}, \ldots, J_{4}$. To calculate $J_{1}$, we use (2.5) to get

$$
\begin{aligned}
\mathbb{E}\left|J_{1}\right|^{q} & \leq C(q)\|h\|_{L^{\infty}\left([0, t+\delta] ; L^{q}\left(\mathbb{R}^{d} \times \Omega\right)\right)}^{q}\left|\int_{t}^{t+\delta}\left[\int_{\mathbb{R}^{d}}|p(t+\delta-r, y)|^{\frac{q}{q^{-1}}} d y\right]^{\frac{(q-1)}{q}} d r\right|^{q} \\
& \leq C(q)\|h\|_{L^{\infty}\left([0, t+\delta] ; L^{q}\left(\mathbb{R}^{d} \times \Omega\right)\right)}^{q}\left|\int_{0}^{\delta} r^{-\frac{d}{q \alpha}} d r\right|^{q} \\
& \leq C(q, \alpha, d)\|h\|_{L^{\infty}\left([0, t+\delta] ; L^{q}\left(\mathbb{R}^{d} \times \Omega\right)\right)}^{q} \delta^{q-\frac{d}{\alpha}} .
\end{aligned}
$$

An analogue calculation also implies that

$$
\begin{aligned}
\mathbb{E}\left|J_{3}\right|^{q} & \leq C(q)\|f\|_{L^{\infty}\left([0, t+\delta] ; L^{q}\left(\mathbb{R}^{d} \times \Omega\right)\right)}^{q}\left|\int_{t}^{t+\delta}\left[\int_{\mathbb{R}^{d}}|p(t+\delta-r, y)|^{\frac{q}{q-1}} d y\right]^{\frac{2(q-1)}{q}} d r\right|^{\frac{q}{2}} \\
& \leq C(q)\|f\|_{L^{\infty}\left([0, t+\delta] ; L^{q}\left(\mathbb{R}^{d} \times \Omega\right)\right)}^{q}\left|\int_{0}^{\delta} r^{-\frac{2 d}{q \alpha}} d r\right|^{\frac{q}{2}} \\
& \leq C(q, \alpha, d)\|f\|_{L^{\infty}\left([0, t+\delta] ; L^{q}\left(\mathbb{R}^{d} \times \Omega\right)\right)}^{q} \delta^{\frac{q}{2}-\frac{d}{\alpha}} .
\end{aligned}
$$

For $J_{2}$, we use Lemma 2.1 (2.3), one concludes that

$$
\begin{aligned}
\mathbb{E}\left|J_{2}\right|^{q} & \leq \mathbb{E}\left|\int_{0}^{t}\right| P_{t+\delta-r} h(r, x)-P_{t-r} h(r, x)|d r|^{q} \\
& \leq\|h\|_{L^{\infty}\left([0, t+\delta] ; L^{\prime}\left(\mathbb{R}^{d} \times \Omega\right)\right)}^{q} \delta^{\beta q}\left|\int_{0}^{t}(t-r)^{-\beta-\frac{d}{q \alpha}} d r\right|^{q} \\
& \leq C(q, \alpha, d, \beta, t)\|h\|_{L^{\infty}\left([0, t+\delta] ; L^{q}\left(\mathbb{R}^{d} \times \Omega\right)\right)}^{q} \delta^{\beta q} .
\end{aligned}
$$

Similarly, one gains

$$
\begin{aligned}
\mathbb{E}\left|J_{4}\right|^{q} & \leq \mathbb{E}\left|\int_{0}^{t}\right| P_{t+\delta-r} f(r, x)-\left.\left.P_{t-r} f(r, x)\right|^{2} d r\right|^{\frac{q}{2}} \\
& \leq C(q)\|f\|_{L^{\infty}\left([0, t+\delta] ; L^{q}\left(\mathbb{R}^{d} \times \Omega\right)\right)}^{q} \delta^{\beta q}\left|\int_{0}^{t}(t-r)^{-2 \beta-\frac{2 d}{q \alpha}} d r\right|^{\frac{q}{2}} \\
& \leq C(q, \alpha, d, \beta, t)\|f\|_{L^{\infty}\left([0, t+\delta] ; L^{q}\left(\mathbb{R}^{d} \times \Omega\right)\right)}^{q} \delta^{\beta q} .
\end{aligned}
$$

Combining (3.4)-(3.7), one arrives at

$$
\begin{aligned}
& \mathbb{E}|u(t+\delta, x)-u(t, x)|^{q} \\
& \quad \leq C(q, \alpha, d, \beta, t)\left[\|h\|_{L^{\infty}\left([0, t+\delta] ; L^{q}\left(\mathbb{R}^{d} \times \Omega\right)\right)}^{q}+\|f\|_{L^{\infty}\left([0, t+\delta] ; L^{q}\left(\mathbb{R}^{d} \times \Omega\right)\right)}^{q}\right]\left[\delta^{q-\frac{d}{\alpha}}+\delta^{\beta q}\right],
\end{aligned}
$$


which implies

$$
\begin{aligned}
& \mathbb{E}|u(t+\delta, x)-u(t, x)|^{q} \\
& \quad \leq C(q, \alpha, d, \beta, t)\left[\|h\|_{L^{\infty}\left([0, t+\delta] ; L^{q}\left(\mathbb{R}^{d} \times \Omega\right)\right)}^{q}+\|f\|_{L^{\infty}\left([0, t+\delta] ; L^{q}\left(\mathbb{R}^{d} \times \Omega\right)\right)}^{q}\right] \delta^{\beta q}
\end{aligned}
$$

if $\delta<1$.

Therefore, we accomplish from (3.3) and (3.8) that

$$
\begin{aligned}
& \mathbb{E}\left|u\left(t_{2}, x_{2}\right)-u\left(t_{1}, x_{1}\right)\right|^{q} \\
& \leq C(q, \alpha, d, \beta)\left[\|h\|_{L^{\infty}\left(\left[0, t_{2}\right] ; L^{q}\left(\mathbb{R}^{d} \times \Omega\right)\right)}^{q}+\|f\|_{L^{\infty}\left(\left[0, t_{2}\right] ; L^{q}\left(\mathbb{R}^{d} \times \Omega\right)\right)}^{q}\right] \\
& \quad \times\left(\left|t_{2}-t_{1}\right|+\left|x_{2}-x_{1}\right|\right)^{\beta q}
\end{aligned}
$$

for every $x_{1}, x_{2} \in \mathbb{R}^{d}, 0 \leq t_{1} \leq t_{2} \leq 1$. According to (3.1) and (3.9), (1.6) is true.

Notice that $q>4(d+1) /(1 \wedge \alpha)$ and (3.9) holds for every $0<\beta<(1 / 2-d /(q \alpha))(1 \wedge \alpha)$. For a given sufficiently large natural number $0<m \in \mathbb{N}$, if one obtains

$$
\beta=\frac{m}{1+m}\left(\frac{1}{2}-\frac{d}{q \alpha}\right)(1 \wedge \alpha)
$$

then

$$
q \beta=q \frac{m}{1+m}\left(\frac{1}{2}-\frac{d}{q \alpha}\right)(1 \wedge \alpha)>\frac{m}{1+m}(d+2)>d+1 .
$$

In view of (3.9) and (3.11), by using Kolmogorov's theorem, $u$ has a continuous version. It remains to prove the Hölder estimate $(1.7)$ on $[0,1]^{d+1}$, and for writing simplicity, we set

$$
A=\|h\|_{L^{\infty}\left([0,1] ; L^{q}\left(\mathbb{R}^{d} \times \Omega\right)\right)}+\|f\|_{L^{\infty}\left([0,1] ; L^{q}\left(\mathbb{R}^{d} \times \Omega\right)\right)} .
$$

One introduces a sequence of sets: $\mathcal{S}_{n}=\left\{z \in \mathbb{Z}^{d+1} \mid z 2^{-n} \in(0,1)^{d+1}\right\}, 0<n \in \mathbb{N}$. For an arbitrary $e=\left(e^{1}, \ldots, e^{d+1}\right) \in \mathbb{N} \times \mathbb{Z}^{d}$ such that $\|e\|_{\infty}=\max _{1 \leq i \leq d+1}\left|e^{i}\right|=1$, and every $z, z+e \in \mathcal{S}_{n}$, we define $v_{z}^{n, e}=\left|u\left((z+e) 2^{-n}\right)-u\left(z 2^{-n}\right)\right|$. Then

$$
\mathbb{E}\left|v_{z}^{n, e}\right|^{q} \leq C(q, \alpha, d) A^{q} 2^{-n \beta q} .
$$

For any $\tau>0$ and $K>0$, one sets a number of events $\mathcal{A}_{z, \tau}^{n, e}=\left\{\omega \in \Omega \mid v_{z}^{n, e} \geq K \tau^{n}\right\}(z, z+e \in$ $\mathcal{S}_{n}$ ), it yields that

$$
\mathbb{P}\left(\mathcal{A}_{z, \tau}^{n, e}\right) \leq \frac{\mathbb{E}\left|v_{z}^{n, e}\right|^{q}}{K^{q} \tau^{q^{n}}} \leq \frac{C(q, \alpha, d) A^{q} 2^{-n \beta q}}{K^{q} \tau^{q^{n}}} .
$$

Observe that, for each $n$, the total number of the events $\mathcal{A}_{z, \tau}^{n, e}\left(z, z+e \in \mathcal{S}_{n}\right)$ is not greater than $2^{(d+1) n} 3^{d+1}$. Hence the probability of the union $\mathcal{A}_{\tau}^{n}=\bigcup_{z, z+e \in S_{n}}\left(\bigcup_{\|e\|_{\infty}=1} \mathcal{A}_{z, \tau}^{n, e}\right)$ meets the estimate

$$
\mathbb{P}\left(\mathcal{A}_{\tau}^{n}\right) \leq C(q, \alpha, d) A^{q} \frac{2^{-n \beta q}}{K^{q} \tau^{q^{n}}} 2^{(d+1) n} \leq C(q, \alpha, d) A^{q} K^{-q}\left(\frac{2^{(d+1)}}{\left(2^{\beta} \tau\right)^{q}}\right)^{n} .
$$


For $m>0$ large enough (given in (3.10)), one takes $\beta$ by (3.10), $\tau=2^{-\beta / m}$, then the probability of the event $\mathcal{A}=\bigcup_{n \geq 1} \mathcal{A}_{\tau}^{n}$ can be calculated as follows:

$$
\mathbb{P}(\mathcal{A}) \leq C(q, \alpha, d) A^{q} K^{-q} .
$$

For every point $\xi=(t, x) \in(0,1)^{d+1}$, we have $\xi=\sum_{i=0}^{\infty} e_{i} 2^{-i}\left(\left\|e_{i}\right\|_{\infty} \leq 1\right)$. Denote $\xi_{k}=$ $\sum_{i=0}^{k} e_{i} 2^{-i}\left(\xi_{0}=0\right)$. For any $\omega \bar{\in} \mathcal{A}$, we have $\left|u\left(\xi_{k+1}\right)-u\left(\xi_{k}\right)\right|<K \tau^{k+1}$, which suggests that

$$
|u(t, x)| \leq \sum_{k=0}^{\infty}\left|u\left(\xi_{k+1}\right)-u\left(\xi_{k}\right)\right|<K \sum_{k=1}^{\infty} \tau^{k}=K \frac{\tau}{1-\tau} \leq K\left(2^{\frac{\beta}{m}}-1\right)^{-1} .
$$

Set $v_{1}=\sup _{(t, x) \in(0,1)^{d+1}}|u(t, x)|$, then $v_{1}=\sup _{(t, x) \in[0,1]^{d+1}}|u(t, x)|$ since $u$ has a continuous version. For any $0<\gamma<q$, it yields that

$$
\begin{aligned}
\mathbb{E} v_{1}^{\gamma} & =\gamma \int_{0}^{\infty} r^{\gamma-1} \mathbb{P}\left(v_{1} \geq r\right) d r \\
& =\gamma \int_{0}^{c K} r^{\gamma-1} \mathbb{P}\left(v_{1} \geq r\right) d r+\gamma \int_{c K}^{\infty} r^{\gamma-1} \mathbb{P}\left(v_{1} \geq r\right) d r .
\end{aligned}
$$

If one chooses $c \geq\left(2^{\frac{\beta}{m}}-1\right)^{-1}$, according to (3.12) and (3.13), from (3.14) one finishes at

$$
\mathbb{E} v_{1}^{\gamma} \leq(c K)^{\gamma}+C(q, \alpha, d) A^{q} \gamma \int_{c K}^{\infty} r^{\gamma-1-q} d r \leq(c K)^{\gamma}+C(q, \alpha, d) A^{q} \gamma K^{\gamma-q},
$$

which hints that

$$
\mathbb{E} v_{1}^{\gamma} \leq C(q, \alpha, d, \gamma) A^{\gamma}
$$

if one chooses $K=A$.

Let us calculate the Hölder semi-norm of $u$. For a solution of (1.1) and for every $\omega \bar{\in} \mathcal{A}$, inequality (2.17) holds for $\kappa_{n}=K \tau^{n}$. With the help of Lemma 2.5 (2.18), one has

$$
|u((t, x)+\Delta)-u(t, x)| \leq 2 K \tau^{-1}|\Delta|^{\log _{2}(1 / \tau)}
$$

for $(t, x),(t, x)+\Delta \in(0,1)^{d+1}$.

Let $\beta$ be described in (3.10). For any $0<\theta<\beta$, if one has $\tau=2^{-\theta}$, we arrive at

$$
|u((t, x)+\Delta)-u(t, x)| \leq 4 K|\Delta|^{\theta},
$$

which hints that

$$
\mathbb{P}\left([u]_{\mathcal{C}^{\theta}\left([0,1]^{d+1}\right)} \geq 4 K\right) \leq \mathbb{P}(\mathcal{A}) \leq C(q, \alpha, d, \theta) A^{q} K^{-q} .
$$

Finally, for any $0<\gamma<q$, analogue calculations of (3.14) and (3.15) imply that

$$
\mathbb{E}\|u\|_{\mathcal{C}^{\theta}\left([0,1]^{d+1}\right)}^{\gamma} \leq C(q, \alpha, d, \gamma, \theta) A^{\gamma} .
$$

From (3.15) and (3.16), and observing that $m \in \mathbb{N}$ is arbitrary, the desired conclusion holds true. 


\section{Acknowledgements}

The authors sincerely thank the referees and the editors for their helpful comments and suggestions.

\section{Funding}

The first author is partially supported by the Fundamental Research Funds for the Central Universities (WUT: 193114001). The third author is partially supported by National Science Foundation of China (11501577).

\section{Competing interests}

The authors declare that they have no competing interests.

\section{Authors' contributions}

All authors carried out the proofs and conceived the study. All authors read and approved the final manuscript.

\section{Author details}

'Department of Statistics, College of Science, Wuhan University of Technology, Wuhan, China. ${ }^{2}$ School of Data Science and Information Engineering, Guizhou Minzu University, Guizhou, China. ${ }^{3}$ School of Statistics and Mathematics, Zhongnan University of Economics and Law, Wuhan, China.

\section{Publisher's Note}

Springer Nature remains neutral with regard to jurisdictional claims in published maps and institutional affiliations.

Received: 29 March 2018 Accepted: 14 April 2019 Published online: 25 April 2019

\section{References}

1. Adams, A., Fourier, J.F.: Sobolev Space. Pure and Applied Mathematics Series, vol. 140. Elsevier, Amsterdam (2005)

2. Applebaum, D.: Lévy Processes and Stochastic Calculus. Cambridge University Press, Cambridge (2009)

3. Bogdan, K., Jakubowski, T.: Estimates of heat kernel of fractional Laplacian perturbed by gradient operators. Commun. Math. Phys. 271(1), 179-198 (2007)

4. Chang, T., Lee, K.: On a stochastic partial differential equation with a fractional Laplacian operator. Stoch. Process. Appl. 122(9), 3288-3311 (2012)

5. Denis, L., Matoussi, A., Stoica, L.: $L^{p}$ estimates for the uniform norm of solutions of quasilinear SPDEs. Probab. Theory Relat. Fields 133(133), 437-463 (2005)

6. Hsu, E., Wang, Y., Wang, Z.: Stochastic De Giorgi iteration and regularity of stochastic partial differential equations. Ann. Probab. 45(5), 2855-2866 (2017)

7. Kim, I., Kim, K.H.: An $L^{p}$-theory of a class of stochastic equations with the random fractional Laplacian driven by Lévy processes. Stoch. Process. Appl. 122(12), 3921-3952 (2012)

8. Kim, K.H.: $L_{q}\left(L_{p}\right)$ theory and Hölder estimates for parabolic SPDEs. Stoch. Process. Appl. 114(2), 313-330 (2004)

9. Krylov, N.V.: On $L_{p}$-theory of stochastic partial differential equations in the whole space. SIAM J. Math. Anal. 27(2), 313-340 (1996)

10. Krylov, N.V.: An analytic approach to SPDEs. Stoch. Partial Differ. Equ.: Six Perspect. 64, 185-242 (1999)

11. Krylov, N.V.: SPDEs in $L^{q}\left(0, \tau, L^{p}\right)$ spaces. Electron. J. Probab. 5(13), 1-29 (2000)

12. Kuksin, S.B.: A stochastic nonlinear Schrödinger equation I: a priori estimates. Proc. Steklov Inst. Math. 225, 219-242 (1999)

13. Kuksin, S.B., Nadirashvili, N.S., Piatnitski, A.L.: Hölder estimates for solutions of parabolic SPDEs. Theory Probab. Appl. 47(1), 152-159 (2003)

14. LV, G., Gao, H., Wei, J., Wu, J.: BMO and Morrey-Campanato estimates for stochastic convolutions and Schauder estimates for stochastic parabolic equations. J. Differ. Equ. 266, 2666-2717 (2019)

15. Pardoux, É.: Stochastic Partial Differential Equations. Lecture Notes Given in Fudan (2007)

16. Rozovskii, B.L.: Stochastic Evolution Systems. Springer, Netherlands (1990)

17. Stein, E.: Singular Integrals and Differentiability Properties of Functions, vol. 30. Princeton University Press, Princeton (1970)

18. Wei, J., Duan, J., Lv, G.: Schauder estimates for stochastic transport-diffusion equations with Lévy processes. J. Math. Anal. Appl. 474, 1-22 (2019)

19. Xie, L.: Singular SDEs with critical non-local and non-symmetric Lévy type generator. Stoch. Process. Appl. 127 3792-3824 (2017)

20. Xie, X., Duan, J., Li, X., Lv, G.: A regularity result for the nonlocal Fokker-Planck equation with Ornstein-Uhlenbeck drift (2015) arXiv:1504.04631 